\title{
Advocating for Equity in Cancer Care
}

James McCanney, JDa; Terrell Johnson, MPA“; Lindsey A.M. Bandini, MPH'; Shonta Chambers, MSW ${ }^{\mathrm{b}}$; Lynette Bonar, RNc; and Robert W. Carlson, $\mathrm{MD}^{\mathrm{a}}$

\section{ABSTRACT}

Demographic factors such as race, socioeconomic status, gender identity, area of residence, native language, and cultural barriers have an effect on outcomes in cancer care. To identify unmet needs, challenges, and opportunities in achieving high-quality, patientcentered cancer care for all, NCCN conducted a yearlong environmental scan, which involved stakeholder meetings with patients and patient advocacy groups to discuss these topics. The findings from this scan informed the corresponding NCCN Patient Advocacy Summit: Advocating for Equity in Cancer Care, held in Washington, DC, on December 10, 2018. In addition to the many patient advocacy groups, the summit featured a number of other stakeholders that advocate for equity in cancer care. This article encapsulates the findings of the environmental scan and the discussion from the NCCN Patient Advocacy Summit.

J Natl Compr Canc Netw 2019;17(9):1043-1048 doi: 10.6004/jnccn.2019.7339

\footnotetext{
${ }^{a}$ National Comprehensive Cancer Network, Plymouth Meeting, Pennsylvania bPatient Advocate Foundation, Hampton, Virginia; and 'Tuba City Regional Health Care Corporation, Tuba City, Arizona.
}

How social determinants contribute to individual health and health equity has been the focus of recent research and increasing consensus. In cancer care, health outcomes are influenced by a variety of socioeconomic factors, including race/ethnicity, geographic location, occupation, gender identity, sexual orientation, and insurance coverage. For instance, studies show that the cancer burden for patients increases with declining socioeconomic status due to myriad factors, such as greater exposure to cancer risk factors, reduced likelihood of early detection, and lack of access to highquality healthcare for timely and effective treatment. ${ }^{1}$ To explore these issues, NCCN hosted the 2018 Patient Advocacy Summit: Advocating for Equity in Cancer Care. In preparation for the meeting, NCCN conducted an environmental scan among patients and patient advocacy groups to identify unmet needs, challenges, and opportunities in achieving high-quality, patient-centered cancer care for all. The corresponding summit featured a diverse group of experts in health inequity who highlighted the disparities in access, quality, and outcomes in cancer care and explored potential policy strategies to address barriers preventing equitable access to highquality cancer care.

\section{Overcoming Disparities: A Case Study on the Navajo Nation}

One glaring example of inequity in cancer care occurs on Native American reservations. According to the 2010 Census, there are 560 Native American tribes in the United States, representing 5.2 million people. ${ }^{2}$ Yet, there was not a single oncology practice on Native American land until January 2019. NCCN invited Lynette Bonar, CEO of the Tuba City Regional Health Care Corporation, to give a keynote presentation at the summit. Tuba City Regional Health Care is the sole hospital in the Navajo Nation, which is the largest and most populated Native American reservation. ${ }^{3}$

The Navajo Nation is approximately the size of the state of West Virginia and is home to $>200,000$ Navajo and Hopi people. ${ }^{4}$ On the Navajo Nation, there are 523 abandoned, open uranium mines, 518 of which are within 1 mile of a water source; 266 of the mines have gamma radiation levels 10 times higher than the naturally occurring background gamma level, and 17 of 
those mines are within 200 feet of an occupied home. ${ }^{5}$ Studies have consistently shown that exposure to radiation causes increased cancer rates and mortality. ${ }^{6}$ Uranium contamination is present in $85 \%$ of the homes in the Navajo Nation, and $95 \%$ of Navajo people have higher radiation levels than the average US citizen. ${ }^{7}$ The Environmental Protection Agency has secured funding to clean up and close 219 of those mines, but the process is in early stages. ${ }^{8}$

In addition to the increased exposure to uranium, Navajo people are more likely to be exposed to risk factors that promote worse outcomes than the rest of the US population. ${ }^{4}$ The poverty rate on the Navajo Nation is $43 \%,{ }^{9}$ compared with $12.3 \%$ for the United States. ${ }^{10}$ Alcohol abuse, diabetes, and obesity rates are all higher for Navajo people compared with the rest of the US population. ${ }^{9}$ The unemployment rate for the Navajo people is $61 \%$, and therefore many Navajo patients live on disability and Social Security checks that average $\$ 550$ per month. ${ }^{4}$ Compounding these economic challenges are additional geographic limitations. Until January 2019, Navajo patients had to travel 200 miles to the closest cancer center. ${ }^{4}$ Additionally, Navajo patients still lack access to technology such as telephones and Wi-Fi, hindering patient-provider communication. ${ }^{4}$ Most patients on the Navajo Nation also speak Navajo as a first language, making it more difficult for them to communicate effectively with oncologists, and thereby making them less likely to engage in shared decisionmaking. Furthermore, individuals living on the Navajo Nation frequently lack consistent running water, electricity, and proper refrigeration, which may present challenges to maintaining a healthy diet throughout and following treatment. ${ }^{4}$

The Tuba City Cancer Center is the first oncology practice to open on a Native American Reservation. Most patients living on the Navajo Nation will no longer have to travel hundreds of miles each way for their oncologist visit, and can instead use their money to pay for treatment. However, socioeconomic factors contributing to disparities in care persist. These patients still lack access to the most basic necessities, uranium contamination remains rampant, and unemployment and poverty rates are still high. Opening the Tuba City Cancer Center was a good first step, but increased social and economic support and environmental remediation are needed to ensure equitable cancer care for the Navajo Nation.

\section{Panel Discussion: A Viewpoint on Cancer Equity From Patient Advocates}

Financial toxicity, lack of information dissemination, and historical instances of medical mistrust threaten underserved communities' ability and willingness to participate in cancer prevention, routine cancer treatment, and clinical trials. Additionally, systemic and individual bias, such as racial or gender bias, require solutions from stakeholders beyond the medical community. Ultimately, if not appropriately addressed, barriers to treatment will compound suboptimal outcomes for the communities most in need.

Immunotherapies and precision-based care signal a new chapter in the field of oncology, providing patients with innovative treatments that reduce toxicities and increase efficacy. However, advocates have identified tertiary expenses associated with treatment as a barrier for underresourced patient populations. Increasingly, transportation costs and loss of wages due to a cancer diagnosis have become significant barriers for many subpopulations. ${ }^{11}$ Similarly, patient advocates have expressed concern that combination therapies, requiring various specialists and diverse care teams, may prevent patients with less access to health resources from actively engaging in their own care. ${ }^{11}$

In addition to challenges tied to treatment costs and financial literacy, equity in cancer care is further threatened by a lack of data operationalization, because vital screening and treatment data fail to get dispensed to communities most in need..$^{12}$ For example, due to increased detection of young-onset colorectal cancer, the American Cancer Society (ACS) recently issued a recommendation to lower the colorectal cancer screening age to 45 years. ${ }^{13}$ However, few insurance companies have adjusted their coverage packages to meet this recommendation. ${ }^{12}$ This inability to operationalize resources and data for colorectal cancer may disproportionately affect African Americans, who are 4 times more likely to develop the disease and 3 times more likely to die of it than their white counterparts. ${ }^{12}$

In the United States, approximately $3 \%$ of people diagnosed with cancer participate in clinical trials, with lower rates reported for women and minority populations. ${ }^{14}$ Moreover, individuals with non-European ancestries are underrepresented in genetic databases, creating incomplete genetic profiles and limiting the ability to apply genetic knowledge toward advancing treatment options. ${ }^{15}$ Historical anecdotes of medical mistrust still permeate underresourced communities, which may contribute to the avoidance of cancer screening and reduced participation in clinical trials. African Americans, in particular, are still acutely aware of medical ethics violations, such as the Tuskegee Syphilis Experiment. ${ }^{16}$ Considering the impact of these historical violations of the patientprovider relationship is important when engaging with African American communities. ${ }^{17}$ Similarly, Native 
Americans have a natural distrust of clinical trials due to historically poor treatment at the hands of the US government. ${ }^{18}$

\section{Patient Advocacy Resources}

In an effort to highlight the patient voice, NCCN convened a series of stakeholder meetings with various patient advocacy groups to explore equitable access to high-quality, effective, and efficient oncology care. To highlight the range of resources that the patient advocacy community provides for underserved individuals with cancer, 6 advocacy groups presented barriers, accomplishments, and future strategies to promote equitable cancer care (Table 1).

\section{Improving Cancer Care With the 4R Oncology Program}

Achieving positive outcomes in cancer treatment requires that the right patients receive the right information and care at the right time and in the right sequence. ${ }^{19}$ To this end, the $4 \mathrm{R}$ Oncology Program was created by the Center for Business Models in Healthcare and collaboratively piloted with Northwestern University to achieve improved outcomes and more guideline-concordant care. ${ }^{20}$

First, the 4R Oncology Program asks physicians to identify individual needs unique to each patient, ranging from treatment type to preferred patient outcome to level of tolerable toxicity. Next, the patient must be given the right information in a timely manner. This step is vital to ensure that each patient can be an active participant in the shared decisionmaking process. Some studies have found that shared decision-making can improve patient satisfaction and promote superior outcomes in both the short and long term. ${ }^{21}$ Technological improvements have enabled patients to access the tools necessary to become active participants in their cancer journey. However, this has also widened the gap between those with means to access the information and those without. ${ }^{19}$ The 4R Oncology Program can bridge that gap and offer a way for oncologists to ensure the right information gets to their patients in language they can understand. ${ }^{19}$

Cancer care is an interdependent process. In many cases, timing of care delivery is just as important as the treatment itself. If timing and sequencing are not effectively managed, deadly delays and breakdowns in care can occur. ${ }^{19}$ The $4 \mathrm{R}$ Oncology Program leverages the clinical expertise of the NCCN Clinical Practice Guidelines in Oncology (NCCN Guidelines) to create a project plan unique to each patient. For example, the 4 R Oncology Program can determine whether a patient has a hereditary risk of breast cancer and needs to be referred to genetic counseling and to potentially undergo biomarker testing, the results of which may help inform appropriate treatment options. However, if the patient did not receive the referral, the testing was never performed. Genetic testing may be challenging to obtain before surgery but after diagnosis. Therefore, utilization of the 4R Oncology Program can ensure that this problem is avoided and that no breakdowns in care occur. ${ }^{19}$

Patients who are most at risk of breakdowns in time and sequence are those previously identified as receiving inequitable care. ${ }^{19}$ In recognition of that fact, the $4 \mathrm{R}$ Oncology Program has been piloted in safety-net hospitals in addition to a large academic cancer center. Initial results demonstrate that the program reduces timing and sequencing errors across patient populations. ${ }^{19}$ In addition, $85 \%$ of patients from the safety-net hospital pilot found the program to be useful compared with $75 \%$ from community and academic hospitals. ${ }^{20}$ In 2019, the program will expand to 11 additional centers, 4 of which serve primarily a low-income patient population. ${ }^{19}$ Projects such as the 4R Oncology Program that leverage clinical guideline expertise with unique tools to promote guideline adherence can effectively decrease inequities in oncology care. ${ }^{22}$

\section{Panel Discussion: Identifying and Addressing the Barriers to Equity in Oncology}

Bias can exist throughout the cancer care continuum; from initial intake through survivorship, practitioners and healthcare systems make assumptions about patients. These assumptions can focus on a patient's age, literacy, sex/gender, race, or even technology competence. Significant evidence shows that biases may affect the care an individual receives. ${ }^{23}$ For example, $90 \%$ of LGB patients surveyed in the emergency department would be willing to respond to questions about sexual orientation and gender identity, in contrast to only $77 \%$ of emergency department providers who indicated they would feel comfortable enough to ask. ${ }^{24}$ This may influence treatment options by hindering patient-provider communication of important issues, such as caregiver support. Although physicians may view these details as personal or unrelated to care, perceived support networks may influence the treatments offered to each individual patient ${ }^{25}$; patients who do not have a spouse or domestic partner are less likely to be offered aggressive treatment, affecting overall outcomes for these patients. ${ }^{26}$

A challenging dichotomy exists between maintaining patient-centered care and reducing the variability of care 


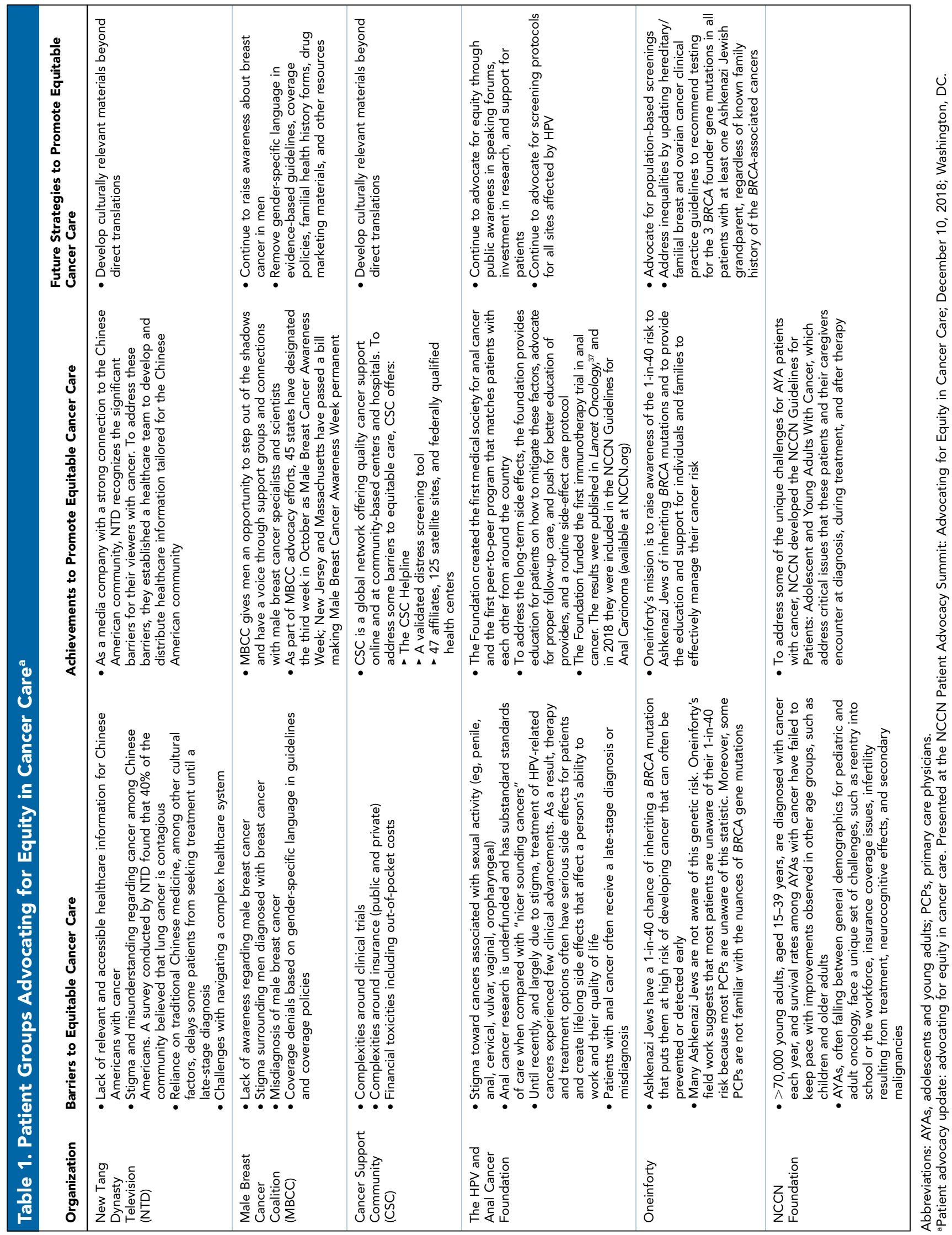


between patients. Researchers have been educated to acknowledge bias in an effort to mitigate its impact on results. Incorporating this practice into medical school curricula is one way to reduce physician bias. ${ }^{27}$ Additionally, medical schools need to address the systemic bias within the healthcare system. ${ }^{28}$ For example, the Supportive Oncology Collaborative demonstrated a straightforward method of addressing a common misconception that all patients can read and write. The initiative worked with key stakeholders to create a combined screening tool for patients with cancer that, beyond the standard measures, included validated health literacy questions. This tool helped identify individuals who may need assistance with forms or written instructions, the first step in connecting patients with necessary support services.

Geographic disparities in life expectancy among US counties are large and increasing, and rapid advancements in healthcare technology may further influence these disparities. ${ }^{29}$ E-communication between patients and physicians may reduce transportation challenges associated with care delivery. ${ }^{30}$ However, the requisite bandwidth distribution needed to operate e-communication devices is not available throughout the country. ${ }^{31}$ Therefore, the continued use of e-communication may further widen the achievement of improved outcomes.

Advances in healthcare technology extend beyond hardware and infrastructure. Improved technology has also resulted in advances in testing and treatment. Biomarker testing is evolving at a rapid pace, and recently several therapies targeting genetic mutations rather than cancer type received FDA approval. ${ }^{27}$ Physicians must remain vigilant in ordering relevant tests for targeted therapies, which is particularly burdensome for smaller community practices that treat a variety of cancer types. ${ }^{32}$ Clinical trial enrollment is becoming increasingly dependent on genetic screening; therefore, these initial tests may also have implications throughout the care continuum. ${ }^{27}$ Discouraging disparities that may impact enrollment criteria is especially important for underrepresented populations. ${ }^{33}$

Conversely, new technology harnesses the potential to improve care in rural communities, eliminating many of the disparities that exist. Practically speaking, advancements in the availability of and reimbursement for telemedicine provide opportunities for remote access to high-level resources that were previously limited to large medical centers, including specialized care plans, supportive services, and clinical trials. ${ }^{34}$ The importance of this is highlighted by rapid advancements in testing and treatment of certain cancers, ${ }^{35}$ resulting in highly complex and nuanced treatment strategies. To ensure that disparities do not continue to increase in rural areas, improving the technology infrastructure in these communities to promote access to high-level resources should be a public health priority. ${ }^{27}$

Directing additional resources toward eliminating the disparities identified is the best way to enact meaningful change. However, when considering that approach, it is important to acknowledge the great burden physicians already face. Fortunately, supportive care services and social workers can adequately address the disparities. Employing a larger supportive staff is a feasible first step in tackling the enormous issue of inequity. Delegating support staff to community engagement, intake screening, and resource identification have all proven successful in improving patient care. ${ }^{24}$ Additionally, payers and insurers need to begin adequately reimbursing for these services, because they are relatively inexpensive yet extremely important in a patient's cancer experience. ${ }^{36}$

\section{Conclusions}

Ample evidence shows that demographic factors, including age, race, socioeconomic status, gender identity, area of residence, language, and cultural barriers, effect outcomes. The advent of telehealth and rapidly improving technologies will affect disparities in some way. Telehealth may serve to combat inequities by giving patients access to physicians they would not otherwise be able to see; however, it also threatens to widen the already substantial healthcare gap for those who do not have access to telehealth. Systemic and individual biases can also deleteriously influence care for certain patient populations.

Stakeholders across the oncology ecosystem have the tools to combat these injustices, and there is a rising tide of change. In 2019, clinicians at the first-ever cancer center on a Native American reservation treated their first patient. The 4R Oncology Program is expanding to 11 additional cancer centers, 4 of which are in locations with an underserved patient population. No one would argue that the issue of inequity is resolved, however. To continue effecting change, all stakeholders must remain vigilant in identifying the areas where work remains.

Submitted May 28, 20198; accepted for publication July 2, 2019.

Disclosures: The authors have disclosed that they have no financial interests, arrangements, or affiliations with the manufacturers of any products discussed in this article or their competitors.

Correspondence: James McCanney, JD, National Comprehensive Cancer Network, 3025 Chemical Road, Suite 100, Plymouth Meeting, PA 19462. Email: mccanney@nccn.org 


\section{References}

1. Horwitz RI. Equity in cancer care and outcomes of treatment: a different type of cancer moonshot. JAMA 2016;315:1231-1232.

2. United States Census Bureau. The American Indian and Alaska Native Population: 2010. Available at: https://www.census.gov/history/pdf/ c2010br-10.pdf. Accessed July 22, 2019.

3. Horwitz S. U.S. to pay Navajo Nation $\$ 554$ million in largest settlement with single Indian tribe. The Washington Post. September 24, 2014. Available at: https://www.washingtonpost.com/world/national-security/ us-to-pay-navajo-nation-554-million-in-largest-settlement-with-singleindian-tribe/2014/09/24/4dc02cc6-434e-11e4-9a15-137aa0153527_ story.html?utm_term $=.25 \mathrm{~d} 2 \mathrm{f} 1 \mathrm{db} 8 \mathrm{e} 14$.

4. Bonar L. Overcoming disparities: a case study on the Navajo Nation. Presented at the NCCN Patient Advocacy Summit: Advocating for Equity in Cancer Care; December 10, 2018; Washington, DC.

5. Hoover J, Gonzales M, Shuey $C$, et al. Elevated arsenic and uranium concentrations in unregulated water sources on the Navajo Nation, USA. Expo Health 2017;9:113-124.

6. Ritz B. Radiation exposure and cancer mortality in uranium processing workers. Epidemiology 1999;10:531-538.

7. Spanne A. Uranium pervades homes on and near Navajo Nation. High Country News. August 27, 2017. Available at: https://www.hcn.org/ articles/pollution-epa-budget-cuts-threaten-to-slow-uraniumcleanup-at-navajo-nation. Accessed August 13, 2019.

8. Cleaning Up Abandoned Uranium Mines. U.S. Environmental Protection Agency. Available at: https://www.epa.gov/navajo-nation-uraniumcleanup/cleaning-abandoned-uranium-mines. Accessed February 5, 2019.

9. Navajo Nation Demographics. Navajo Community Development Financial Institution. Available at: http://navajobusiness.com/fastFacts/ Overview.htm. Accessed March 12, 2019

10. Fontenot K, Semega J, Kollar M. Income and Poverty in the United States: 2017. Washington, DC: US Census Bureau; 2018. P60-263.

11. Cryer D. A viewpoint on cancer equity from patient advocates. Panel discussion at the NCCN Patient Advocacy Summit: Advocating for Equity in Cancer Care; December 10, 2018; Washington, DC.

12. Sapienza M. A viewpoint on cancer equity from patient advocates. Panel discussion at the NCCN Patient Advocacy Summit: Advocating for Equity in Cancer Care; December 10, 2018; Washington, DC.

13. Colorectal cancer screening guidelines. American Cancer Society website. Available at: https://www.cancer.org/health-care-professionals/ american-cancer-society-prevention-early-detection-guidelines/ colorectal-cancer-screening-guidelines.html. Accessed February 14, 2019.

14. Dialogues on diversifying clinical trials: successful strategies for engaging women and minorities in clinical trials. Available at: http://www.fda.gov/ downloads/ScienceResearch/SpecialTopics/WomensHealthResearch/ UCM334959.pdf. Accessed March 1, 2019.

15. Smith CE, Fullerton SM, Dookeran KA, et al. Using genetic technologies to reduce rather than widen, health disparities. Health Aff (Millwood) 2016;35:1367-1373.

16. Scharff DP, Matthews KJ, Jackson P, et al. More than Tuskegee: understanding mistrust about research participation. J Health Care Poor Underserved 2010;21:879-897.

17. Foster W. A viewpoint on cancer equity from patient advocates. Panel discussion at the NCCN Patient Advocacy Summit: Advocating for Equity in Cancer Care; December 10, 2018; Washington, DC.

18. Bonar L. A viewpoint on cancer equity from patient advocates. Panel discussion at the NCCN Policy Summit: Advocating for Equity in Cancer Care; December 10, 2018; Washington, DC.

19. Trosman J. "Raising All Boats" with the 4R Oncology Program: improving cancer care for the underserved. Presented at the NCCN Patient Advocacy Summit: Advocating for Equity in Cancer Care; December 10, 2019; Washington, DC.
20. Weldon CB, Trosman JR, Perez CB, et al. Results from a pilot of an innovative 4R Cancer Care Delivery Model in early breast cancer: impact on timing and sequencing of guideline recommended care [abstract]. J Clin Oncol 2018;36(Suppl):Abstract 561.

21. Hack TF, Degner LF, Watson $P$, et al. Do patients benefit from participating in medical decision-making? Longitudinal follow-up of women with breast cancer. Psychooncology 2006;15:9-19.

22. Dans $A M$, Dans $L, O x m a n A D$, et al. Assessing equity in clinical practice guidelines. J Clin Epidemiol 2007;60:540-546.

23. Penner L, Dovidio J, Gonzalez R, et al. The effects of oncologist implicit racial bias in racially discordant oncology interactions. J Clin Oncol 2016; 34:2874-2880

24. Haider AH, Schneider EB, Kodadek LM, et al. Emergency department query for patient-centered approaches to sexual orientation and gender identity: the EQUALITY study. JAMA Intern Med 2017;177:819-823.

25. Epstein RM, Duberstein PR, Fenton JJ, et al. Effect of a patient-centered communication intervention on oncologist-patient communication, quality of life and health care utilization in advanced cancer. JAMA Óncol 2017;3:92-100.

26. Feng $Y$, Dai $W, L i$, et al. The effect of marital status by age on patients with colorectal cancer over the past decades: a SEER-based analysis. Intern J of Colorectal Disease 2018;33:1001-1010.

27. Chambers S. Identifying and addressing the barriers to equity in oncology. Panel discussion at the NCCN Patient Advocacy Summit: Advocating for Equity in Cancer Care; December 10, 2018; Washington, DC

28. Dickson N. Identifying and addressing the barriers to equity in oncology. Panel discussion at the NCCN Patient Advocacy Summit: Advocating for Equity in Cancer Care; December 10, 2018; Washington, DC.

29. Dwyer-Lindgren L, Bertozzi-Villa A, Stubbs RW, et al. Inequalities in life expectancy among US counties, 1980 to 2014 : temporal trends and key drivers. JAMA Intern Med 2017;177:1003-1011.

30. DuBenske LL, Gustafson DH, Shaw BR, et al. Web-based cancer communication and decision making systems: connecting patients, caregivers, and clinicians for improved health outcomes. Med Decis Making 2010;30:732-744.

31. Mapping Broadband Health in America 2017. Federal Communications Commission. Available at: https://www.fcc.gov/reports-research/maps/ connect2health/\#II=40,-95\&z=4\&t=insights\&inb $=$ in $b b$ access\&inh $=$ in_diabetes_rate $\& d m f=$ none\&inc $=$ none $\& \mathrm{sl} b=90,100 \& \mathrm{slh}=10,22$.

32. Novel Drug Approvals for 2019. U.S. Food \& Drug Administration website. Available at: https://www.fda.gov/Drugs/DevelopmentApprovalProcess/ Druglnnovation/ucm629491.htm. Accessed February 6, 2019.

33. Chen $\mathrm{C}$, Wong R. Black patients miss out on promising cancer drugs. ProPublica website. Available at: https://www.propublica.org/article/ black-patients-miss-out-on-promising-cancer-drugs. Accessed March 8, 2019.

34. Cox A, Lucas G, Marcu A, et al. Cancer survivors' experience with telehealth: a systemic review and thematic synthesis. J Med Internet Res 2017; 19:e11.

35. DeVito T, Leavitt J, Saleh N. Key advances in oncology, 2018. Oncology (Willison Park) 2019;33:6-8.

36. Weldon $\mathrm{C}$. Identifying and addressing the barriers to equity in oncology. Panel discussion at the NCCN Patient Advocacy Summit: Advocating for Equity in Cancer Care; December 10, 2018; Washington, DC.

37. Morris VK, Salem ME, Nimeiri H, et al. Nivolumab for previously treated unresectable metastatic anal cancer (NC19673): a multicentre, single-arm, phase 2 study. Lancet Oncol 2017;18:446-453. 